\title{
Anesthesia Techniques in Brachytherapy
}

\author{
(D) ilknur YILDIRIM \\ Department of Clinic Oncology-Anesthesiology, İstanbul University, Institute of Oncology, İstanbul-Turkey
}

\section{Introduction}

Brachytherapy plays a significant role in the treatment of various cancers and is a treatment in which radioactive sources are placed in or near the tumor. This treatment modality causes the anesthesiologists to encounter a number of difficulties slightly different from the situations in which they encounter surgical patients in the operating room. The first of these difficulties is the fact that patients who are planned for brachytherapy are already elderly, and that these patients have a high risk of perioperative complications.[1,2] Second, intracavitary brachytherapy and interstitial brachytherapy are extremely painful and require both analgesia and immobilization. The American Brachytherapy Association also recommends the use of conscious sedation for intracavitary high-dose rate (HDR) applications of patients with cervical cancer whenever possible.[3] However, analgesia is usually not required after the removal of the applicator.

The duration of the brachytherapy procedures is highly variable. Imaging of the applicator requires X-ray, computed tomography (CT), and increasingly magnetic resonance imaging (MRI) procedures. Computer-based planning also plays an important part of the entire procedure while the applicator is in place. All these procedures lead to prolongation of each treatment section. It is difficult to manage prolonged procedures in elderly patients and patients with comorbid diseases.

Third, a brachytherapy treatment session occurs in more than one place, i.e., in the operating room, X-ray, radiology units for CT or MR, and brachytherapy for radiotherapy in the radiotherapy room, and it is challenging to apply and monitor general anesthesia in different units. [4] The inadequacy of experienced personnel to follow-up the patient between the postoperative recovery room and the units is another problem.[5]
Different brachytherapy procedures require different anesthesia techniques according to the patient and the available equipment to better deal with these problems. In our study, anesthesia types will be mentioned in brachytherapy applications.

\section{Sedation and Systemic Analgesia}

Sedation can be used as an alternative to general anesthesia in less painful brachytherapy procedures. Inhalation anesthesia with nitrogen protoxides can be used for short-term disturbances for placement or removal of the applicator in the cervical HDR brachytherapy.[6]

In addition, opioids, midazolam, and in prolonged cases propofol and sedoanalgesia can be applied. $[7,8]$

Although radiotherapists have a tendency to perform sedation in recent years, this should not be performed without adequate information, equipment, and post-anesthetic care conditions to ensure the patient's safety.

Paracetamol and diclofenac sodium appear to be useful in controlling pain as long as the applicator is on the patient in low-dose rate (LDR) brachytherapy applications.[5]

\section{General Anesthesia}

The primary indications for general anesthesia in brachytherapy are cases where upper body region cancers, such as bronchial, liver, and breast carcinoma, or regional anesthesia techniques are contraindicated. In addition, brachytherapy of the oropharyngeal region tumors in the patient is accompanied by fiberoptic bronchoscopy, and the patient is intubated with general anesthesia. Cases with general anesthesia require complete anesthesia equipment in the field of application.

Although general anesthesia is applied and the application area is in the same place, follow-up of the patient who has received general anesthesia in X-ray, CT, 
MRI, and irradiation room transfers requires considerable attention and experienced health personnel.[5]

\section{Local Anesthesia}

Local anesthesia is widely used by radiotherapists. Jones et al. [9] compared topical lidocaine to placebo for gynecological brachytherapy and showed significantly lower pain in the lidocaine group. However, moderate pain still persists even in the topical lidocaine group.

Generally, topical or local anesthesia is less effective than other anesthesia techniques. Owing to the lack of anesthesiology staff, brachytherapy is still under local anesthesia by radiotherapists wherever possible. However, insufficient analgesia may cause a high degree of discomfort for patients and may lead to interruption of the brachytherapy procedure due to stress-induced movements.

\section{Regional Anesthesia}

Regional anesthesia technique offers advantages for brachytherapy of the lower body parts. It provides adequate analgesia and immobilization, facilitates the transfer of patients between units, and is less risky than general anesthesia. Spinal anesthesia can be used in brachytherapy for patients with gynecological, urologi$\mathrm{cal}$, and lower rectal cancer. A fast start and well-known anesthesia time are its main advantages. Saddle block, a type of spinal anesthesia, may be sufficient in patients treated for prostate or anal cancer. This block is an application in which the local anesthesia is given to the spinal space at low volume, and the block is restrained in the perianal region by sitting for a period of time. However, since immobilization is not fully achieved, applicators are at risk of dislocation during patient transfer.[10]

A lumbar epidural block can be used as an alternative to spinal anesthesia, but the onset of effect is longer than spinal anesthesia. It is used together with a catheter technique or as a caudal epidural block. A caudal block can be used in gynecological brachytherapy and is well tolerated by patients. Application in elderly patients is technically difficult.[11]

A catheter or combined techniques (epidural and spinal catheters or combined spinal-epidural techniques) provide the extension of anesthesia for pulsedose rate (PDR)/LDR brachytherapy if necessary or for adequate analgesia.[4,12] In addition, spinal catheters offer careful dose titration in high risk patients, thereby minimizing hemodynamic side effects.[12] Continuous epidural infusion or epidural patient-controlled analgesia is useful for both LDR and provides lower total local anesthetic dose. Epidural anesthesia can also be used safely in children.[13,14] The lack of experienced staff and the inability to monitor the patient for
$24 \mathrm{~h}$ may limit the use of epidural analgesia for LDR/ PDR. The impossibility of controlling the catheter insertion when full immobilization is mandatory may also impede the use of this technique.

Some researchers have retrospectively compared patients who underwent regional or general anesthesia for brachytherapy applications in patients with cervical carcinoma with the hypothesis that regional anesthesia may affect the sympathetic nervous system, thereby reducing lymphatic flow and, therefore, may have a positive effect on tumor recurrence. However, the effect of anesthesia on tumor progression was not shown.[15]

\section{Other Techniques}

The above-mentioned techniques may be administered or, alternatively, a bilateral dorsal penile block may be made in the case of penile cancers.[16] Owing to the risk of pneumothorax in breast brachytherapy, an intercostal block is rarely applied.

Prostate brachytherapy applications with pudendal nerve block have also been reported.[17]

Sedation-local anesthesia combination or general anesthesia can be applied in lower lip carcinomas, but it can be applied with mental block.[18]

Brachytherapy applications have been reported with hypnosedation, albeit in rare cases.[19]

\section{How Do We Decide?}

Although the anesthetic methods that can be used in brachytherapy applications are clearly defined, the lack of a randomized controlled study on which anesthesia method is better is difficult for selection. In clinical practice, the choice of anesthesia technique depends, in particular, on the body area of brachytherapy, whether patients are planned for LDR, HDR, or PDR and local infrastructure, which may differ from the conditions in surgical patients.[20]

In general, anesthesiologists and related equipment are not always available on a regular basis outside the operating room.

Radiotherapists tend to apply brachytherapy with local anesthesia and, in some cases, apply sedation. However, the levels of analgesia are not sufficient according to other methods, and the discomfort that patients feel is generally unknown. Moreover, brachytherapy procedures are becoming more sophisticated, and local anesthesia or sedation is insufficient in these applications. Adequate conditions for anesthesia include not only complete staffing and technical equipment but also post-anesthetic care. Therefore, the application of the right technique with the experienced anesthesia teams under ideal conditions is critical for both the patient and the radiotherapists. 
Peer-review: Externally peer-reviewed.

Conflict of Interest: There is no conflict of interest.

Financial Support: This presentation did not receive any spesific grant.

\section{References}

1. Wollschlaeger K, Connell PP, Waggoner S, Rotmensch J, Mundt AJ. Acute problems during low-dose-rate intracavitary brachytherapy for cervical carcinoma. Gynecol Oncol 2000;76(1):67-72.

2. Petereit DG, Sarkaria JN, Chappell RJ. Perioperative morbidity and mortality of high-dose-rate gynecologic brachytherapy. Int J Radiat Oncol Biol Phys 1998;42(5):1025-31.

3. Nag S, Erickson B, Thomadsen B, Orton C, Demanes JD, Petereit D. The American Brachytherapy Society recommendations for high-dose-rate brachytherapy for carcinoma of the cervix. Int J Radiat Oncol Biol Phys 2000;48(1):201-11.

4. Benrath J, Kozek-Langenecker S, Hüpfl M, Lierz P, Gustorff B. Anaesthesia for brachytherapy--51/2 $\mathrm{yr}$ of experience in 1622 procedures. Br J Anaesth 2006;96(2):195-200.

5. Roessler B, Six LM, Gustorff B. Anaesthesia for brachytherapy. Curr Opin Anaesthesiol 2008;21(4):514-8.

6. Tyrie LK, Hoskin PJ. Intrauterine high dose rate afterloading brachytherapy: experience of fractionated therapy using a cervical sleeve technique. Clin Oncol (R Coll Radiol) 1996;8(6):376-9.

7. Nguyen TV, Petereit DG. High-dose-rate brachytherapy for medically inoperable stage I endometrial cancer. Gynecol Oncol 1998;71(2):196-203.

8. Oei-Lim VL, Kalkman CJ, van Tienhoven G, Engbers $\mathrm{FH}$. Remote controlled prolonged conscious sedation for gynaecological radiotherapy. Anaesthesia 1996;51(9):866-8.

9. Jones B, Tan LT, Blake PR, Dale RG. Results of a questionnaire regarding the practice of radiotherapy for carcinoma of the cervix in the UK. Br J Radiol 1994;67(804):1226-30.
10. Lam ST, Cho PS, Marks RJ 2nd, Narayanan S. Detection and correction of patient movement in prostate brachytherapy seed reconstruction. Phys Med Biol 2005;50(9):2071-87.

11. Wong SY, Li JY, Chen C, Tseng CH, Liou SC, Tsai SC, et al. Caudal epidural block for minor gynecologic procedures in outpatientsurgery. Chang Gung Med J 2004;27(2):116-21.

12. Michalek-Sauberer A, Kozek-Langenecker SA, Heinzl H, Deusch E, Chiari A. Median effective local anesthetic doses of plain bupivacaine and ropivacaine for spinal anesthesia administered via a spinal catheter for brachytherapy of the lower abdomen. Reg Anesth Pain Med 2008;33(1):4-9.

13. Hogan Q. Epidural catheter tip position and distribution of injectate evaluated by computed tomography. Anesthesiology 1999;90(4):964-70.

14. Gustorff B, Lierz P, Felleiter P, Knocke TH, Hoerauf $\mathrm{K}$, Kress HG. Ropivacaine and bupivacaine for longterm epidural infusion in a small child. Br J Anaesth 1999;83(4):673-4.

15. Ismail H, Ho KM, Narayan K, Kondalsamy-Chennakesavan S. Effect of neuraxial anaesthesia on tumour progression in cervical cancer patients treated with brachytherapy: a retrospective cohort study. Br J Anaesth 2010;105(2):145-9.

16. Szmuk P, Ezri T, Ben Hur H, Caspi B, Priscu L, Priscu V. Regional anaesthesia for circumcision in adults: a comparative study. Can J Anaesth 1994;41(12):1181-4.

17. Schenck M, Schenck C, Rübben H, Stuschke M, Schneider T, Eisenhardt A, et al. Pudendal nerve block in HDR-brachytherapy patients: do we really need general or regional anesthesia? World J Urol 2013;31(2):417-21.

18. Hafez O, Ackerman RS, Evans T, Patel SY, Padalia DM. Mental Nerve Blocks for Lip Brachytherapy: A Case Report. A A Pract 2018;10(10):265-6.

19. Chapet O, Udrescu C, Horn S, Ruffion A, Lorchel F, Gaudioz S, et al. Prostate brachytherapy under hypnosedation: A prospectiveevaluation. Brachytherapy 2019;18(1):22-8.

20. Fitz-Henry J, Chan S. Analgesia for pelvic brachytherapy. Br J Anaesth 2002;89(2):342. 